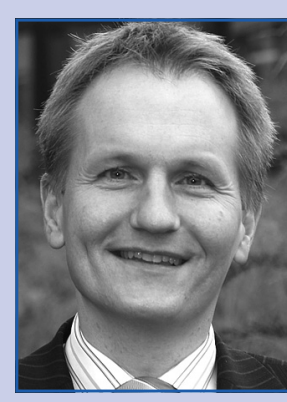

Prof. Dr. Sven Reinecke ist Direktor des Instituts für Marketing an der Universität St. Gallen (HSG) und Leiter des dortigen Kompetenzzentrums "Marketing Performance Management".

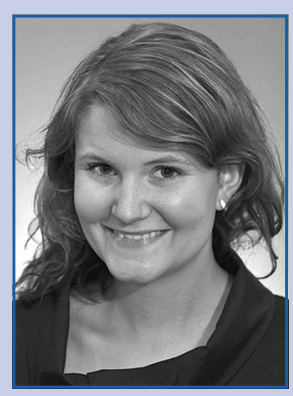

Dipl.-Kffr. Jasmin Eberharter ist wissenschaftliche Mitarbeiterin am Institut für Marketing an der Universität St. Gallen (HSG).
Stichwörter

- Marketingbudgetierung

- Marketingcontrolling

- Marketingcontrolling-Instrumente

a Marketingkennzahlen

- Return on Marketing

\title{
Marketingcontrolling 2010: Einsatz von Methoden und Verfahren des Marketingcontrollings in der Praxis
}

\author{
Sven Reinecke und Jasmin Eberharter
}

Die Relevanz des Themas Marketingcontrolling in der Praxis ist unbestritten: Gerade in wirtschaftlich anspruchsvollen Zeiten müssen Marketingführungskräfte die Wirksamkeit und Wirtschaftlichkeit des Einsatzes des Marketingbudgets nachweisen. Wissenschaftler aus den Bereichen Marketing und Controlling haben insbesondere in den vergangenen Jahren zahlreiche Vorschläge für ein wirkungsvolles Marketingcontrolling entwickelt. Dennoch scheint eine große Diskrepanz zwischen theoretisch-konzeptioneller Entwicklung einerseits und ihrer Umsetzung in der unternehmerischen Praxis andererseits zu bestehen. Der folgende empirische Beitrag untersucht, welche Instrumente und Verfahren des Marketingcontrollings in der Praxis tatsächlich eingesetzt werden. Daraus wird zum einen abgeleitet, in welchen Bereichen noch Implementierungsprobleme existieren; zum anderen wird analysiert, bezüglich welcher Aspekte weiterer Forschungsbedarf im Bereich Marketingcontrolling besteht.

\section{Einleitung}

In der deutschsprachigen Marketingwissenschaft erlebt das Thema Marketingcontrolling nach intensiven Forschungstätigkeiten zu Beginn der achtziger Jahre einen neuen Höhepunkt (vgl. Link/Gerth/ Voßbeck, 2000, Reinecke, 2004, Bauer/ Stokburger/Hammerschmidt, 2006, Reinecke/Janz, 2007). Seitdem das amerikanische Marketing Science Institute das Thema „Marketing Metrics“ mehrfach hintereinander zum Thema mit der höchsten Forschungsrelevanz erhoben hat, ist auch in der internationalen Marketingwissenschaft eine deutlich verstärkte Auseinandersetzung mit diesem Thema zu spüren (vgl. Ambler, 2003, Moorman/Lehmann, 2004, Rust et al., 2004, Farris et al., 2006, O'Sullivan/Abela, 2007, Homburg/Droll/ Tozek, 2008). Der wissenschaftliche Fokus lag dabei einerseits auf der Beschreibung und dem Nachweis der Wirkungskette von der Marketingaktivität bis zum unternehmerischen Erfolg, zum anderen auf der Definition und Auswahl geeigneter Marketingführungsgrößen.

Im Mittelpunkt der nachfolgenden Ausführungen steht die kritische Analyse des aktuellen Status des Marketingcontrollings in der betriebswirtschaftlichen Praxis: Wie hoch ist die Zufriedenheit mit dem Marketingcontrolling? Welches sind die zentralen Herausforderungen, die im Rahmen des Marketingcontrollings zu bewältigen sind? In welchem Ausmaß stehen dem Marketingcontrolling die erforderlichen Ressourcen zur Verfügung, um seinen Aufgaben Rechnung zu tragen? Verlangt die Unternehmensleitung einen Nachweis der Effektivität und Effizienz von Marketingmaßnahmen sowie der Gesamtfunktion „Marketing“? Wie wird das Marketingcontrolling dieser Nachweispflicht gerecht, und welche Instrumente werden genutzt? Zusammengefasst: Wie ist der derzeitige Stand des Marketingcontrollings in der Praxis?

Um diese Fragen zu beantworten, hat das Institut für Marketing an der Universität St. Gallen eine empirische Untersuchung durchgeführt. Nachfolgend wird der Studienaufbau kurz dargestellt, bevor erste Studienergebnisse präsentiert werden.

\section{Anlage der empirischen Studie}

Für die Studie wurden insgesamt 3.904 Manager im deutschsprachigen Raum per E-Mail kontaktiert und gebeten, an einer standardisierten schriftlichen Online-Befragung teilzunehmen. Die Bewertung der Fragen erfolgte zum größten Teil anhand einer 3-stufigen Skala sowie 
einer 7-stufigen Likert Skala. Während des Erhebungszeitraums (Februar/März, 2010) füllten 516 Probanden den Fragebogen aus, so dass die Nettorücklaufquote bei rund 13,0 \% lag. Dies kann als sehr zufrieden stellend bewertet werden.

Die meisten Befragten bekleiden eine leitende Funktion in ihrem Unternehmen: Geschäftsführer, Marketingleiter und Aufsichts- bzw. Verwaltungsräte machen insgesamt 56,5 \% der Befragten aus. Die restlichen $43,5 \%$ setzen sich u. a. aus Verkaufs-, Werbeleitern und Controllern zusammen. 33,4 \% der befragten Unternehmen sind ausschließlich national tätig, 66,6 \% der Unternehmen agieren auf internationalen Märkten. 35 \% der Unternehmen beschäftigen 500 oder mehr Mitarbeiter, $32 \%$ zwischen 50 bis 499 Mitarbeiter und $33 \%$ der Unternehmen beschäftigen weniger als 50 Mitarbeiter. Die Stichprobe umfasst ein breites Branchenspektrum, wobei der Großteil der befragten Unternehmen im Dienstleistungsbereich tätig ist (49,7\%). Die andere Hälfte der Probanden verteilen sich auf die Branchen Industriegüter (19\%), Handel und Distribution (17\%) sowie Konsumgüter (13\%). Die Studie erhebt keinen Anspruch auf vollständige Repräsentativität für den deutschsprachigen Raum, auch wenn die Stichprobenstruktur der mittelständisch geprägten Wirtschaftslandschaft weitgehend entspricht.

\section{Stand des Marketingcontrollings in der Praxis}

Die Notwendigkeit, den „Return on Marketing" (ROM) an das Top-Management zu berichten bzw. die Marketingeffizienz $\mathrm{zu}$ belegen, polarisiert stark. Bei einigen Unternehmen ist sie sehr hoch, bei anderen gar nicht ausgeprägt. Andererseits kann festgestellt werden, dass in den letzten drei Jahren die Effizienz-Nachweispflicht deutlich gestiegen ist (siehe Abb. 1).

Die Marketingleitung schreibt dem Marketing mit großer Mehrheit einen Investitionscharakter zu; das Top-Management der befragten Unternehmen sieht dies jedoch deutlich anders und nimmt Marketing relativ häufig primär als Aufwand wahr. Für das Marketingmanagement kann dies als eindeutige Handlungsaufforderung betrachtet werden: Wenn Marketing eine Wert stiftende Funktion attes-

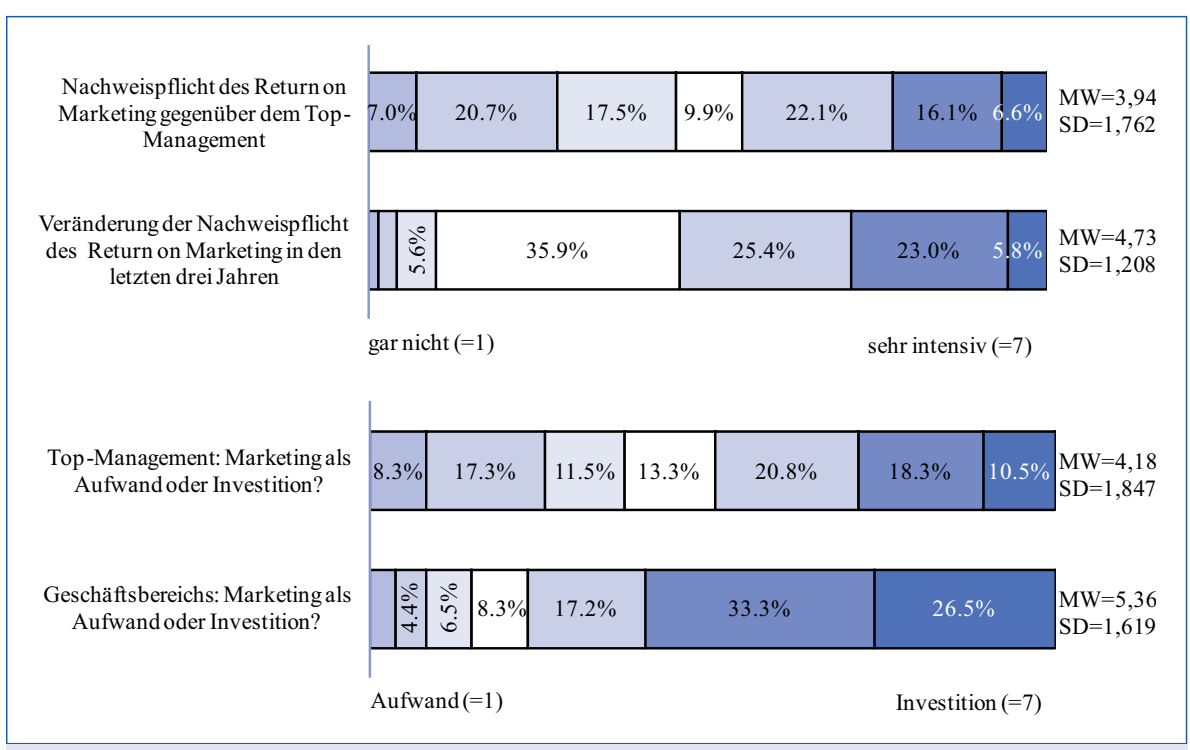

Abb. 1: Marketingwahrnehmung zwischen Aufwand und Investition

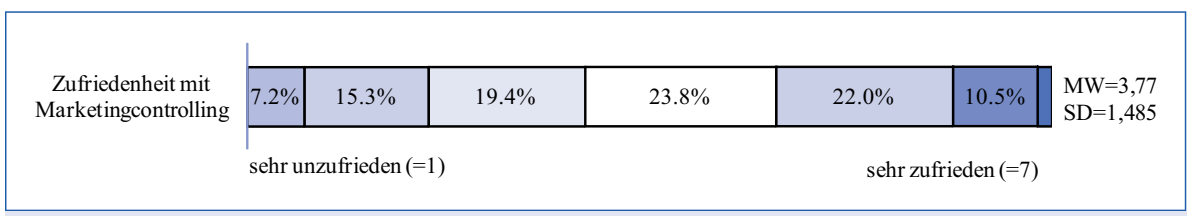

Abb. 2: Zufriedenheit mit dem Marketingcontrolling

tiert werden soll, so muss diese auch (finanzwirtschaftlich) nachgewiesen werden. Dafür ist es erforderlich, geeignete Instrumente zur Erfassung von Effektivität und Effizienz der verschiedenen Marketingmaßnahmen im Unternehmen einzusetzen. Ohne solides Marketingcontrolling fehlt die Basis, um Marketing einen betriebswirtschaftlichen Investitionscharakter zuzuschreiben.

\section{Zufriedenheit mit dem Marketingcontrolling}

Aufgabe des Marketingcontrollings ist es, die Effektivität (Wirksamkeit) und die Effizienz (Wirtschaftlichkeit, Output/InputBetrachtung) des Marketingmanagements sicherzustellen (vgl. Reinecke/Janz, 2007). Die Zufriedenheit mit dem Marketingcontrolling ist allerdings bei kaum einem Unternehmen sehr hoch; vielmehr dominieren tendenziell stärker Unzufriedenheit bzw. Indifferenz (Abb. 2). Insgesamt kann die Zufriedenheit mit dem Marketingcontrolling als sehr mäßig eingeschätzt werden.

Zusammenfassend lässt sich feststellen, dass viele Marketingführungskräfte lediglich behaupten, dass Marketing eine Investition sei - ohne jedoch tatsächlich auf ein zufriedenstellendes Marketingcontrolling zurückgreifen zu können, das in der Lage wäre, einen Wirtschaftlichkeitsnachweis zu erbringen. Die Marketingrealität ist somit von einem wertbzw. investitionsorientierten Marketing noch weit entfernt.

\section{Herausforderungen des Marketingcontrollings}

Welches sind die wesentlichen Ursachen für die Unzufriedenheit mit dem Marketingcontrolling? Worin liegen die größten Herausforderungen?

Die Ergebnisse in Abb. 3 zeigen, dass vor allem die Messbarkeit des Marketingerfolgs, der Aufbau einer hochwertigen Informations- und Datenbasis und das verfügbare Know-how die zentralen Herausforderungen für ein professionelles Marketingcontrolling darstellen. Dabei ist interessant, dass dies alles Bereiche sind, die vom Marketingmanagement selbst maßgeblich beeinflusst werden können. Insbesondere das Know-how ließe sich durch Personalselektion und Weiterbildungsmaßnahmen deutlich verbessern. Ferner kann die Marketingleitung durch eine Differenzierung der Zielplanung sowie die Definition und eindeutige Operationalisierung von Kennzahlen sowohl die Messbarkeit als auch die Informationsbasis im Bereich Marketing selber 


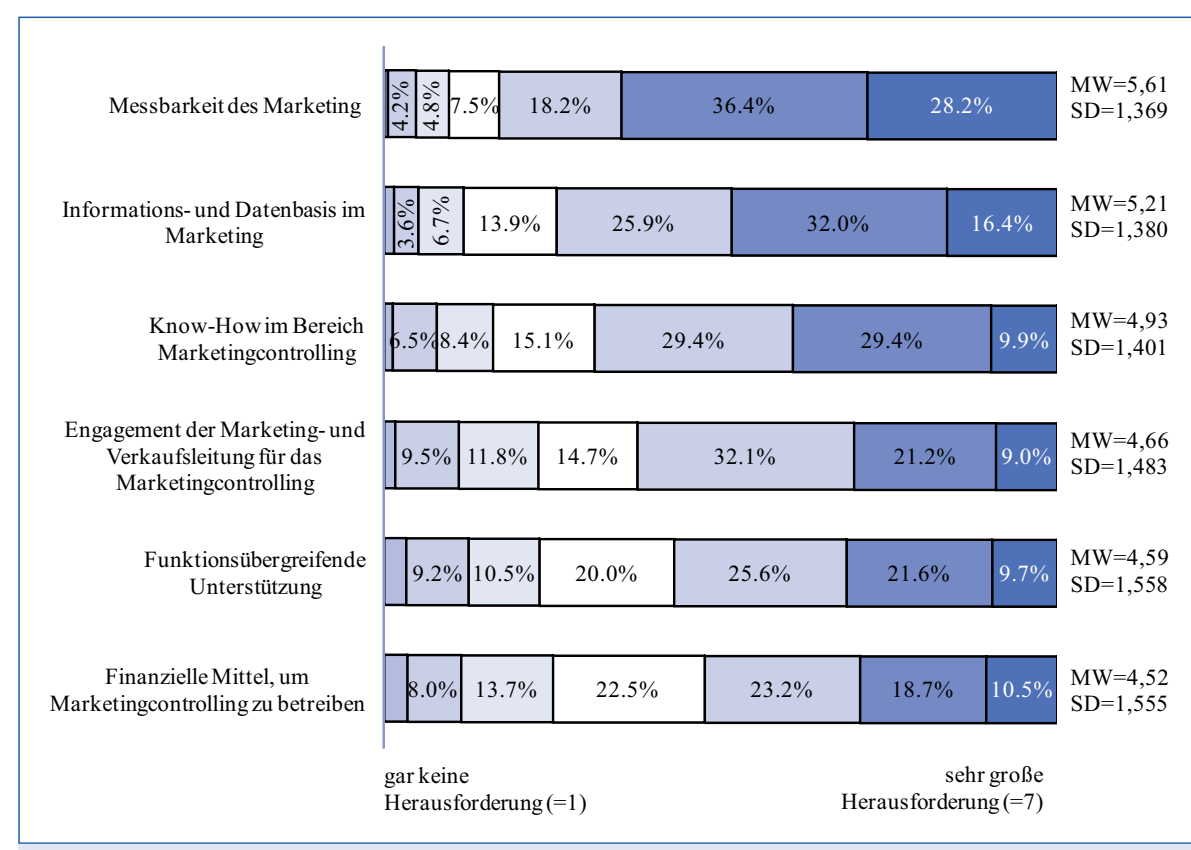

Abb. 3: Herausforderungen des Marketingcontrollings

deutlich verbessern. Eindeutig operationalisierte Marketingziele wären mit Hilfe von Marktforschung und Marketingaccounting problemlos messbar. Somit ist eigentlich nicht die Messbarkeit, sondern vielmehr die eindeutige Zuordnung von Ursache-Wirkungen die dominierende Herausforderung des Marketingcontrollings. Letzteres würde Experimente erfordern, die in der Praxis zumeist an den nicht kontrollierbaren vielfältigen Feldeinflüssen und den damit verbundenen hohen Marktforschungskosten scheitern.

\section{Budgetierung}

Bei der Marketingbudgetierung handelt es sich um eine grundlegende Marketingcontrollingaufgabe, in deren Rahmen Unternehmen sowohl über die Höhe als auch über die Verteilung ihrer für das Marketing einzusetzenden finanziellen Ressourcen zu entscheiden haben. Angesichts ihres hohen Anteils an den Gesamtkosten und ihres schwer zu erfassenden betriebswirtschaftlichen Nutzens stehen Marketingmaßnahmen in vielen Un-

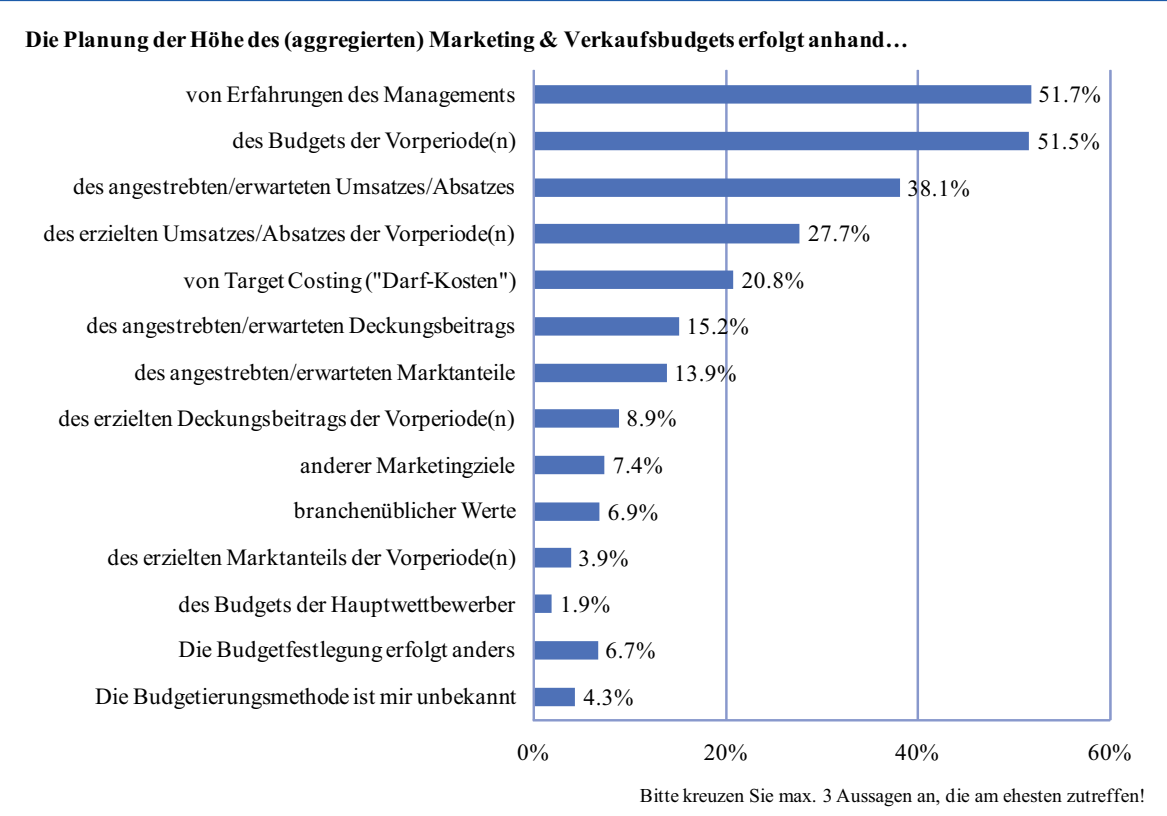

Abb. 4: Marketing- und Verkaufsbudgetierung ternehmen seit einiger Zeit verstärkt im Mittelpunkt von Rationalisierungsprozessen des Top-Managements, das den Ausweis und die Maximierung eines messbaren Beitrags des Marketing zum Unternehmenserfolg bzw. -wert einfordert (vgl. Sheth/Sisodia, 1995, S. 9 f., 2002, S. 349 f.; Weber, 2002, S. 705 f.).

Da Unternehmen in der Regel mehrere Ansätze und Methoden zur Planung der Höhe des (aggregierten) Marketing- und Verkaufsbudgets heranziehen und kombinieren (z. B. Reinecke/Janz, 2007, S. 127 ff.; Fuchs, 2008), wurden die Befragten gebeten, bis zu drei der von ihnen primär für die Marketingbudgetierung genutzten Ansätze und Methoden zu nennen. Wie die in Abb. 4 dargestellten Ergebnisse zeigen, ist die Marketingbudgetierung in der Unternehmenspraxis stark geprägt durch inkrementelle und induktive Verfahren. Grundsätzlich problematisch erscheint, dass die Planung des Marketingbudgets in den meisten Fällen primär auf Basis von Managementerfahrung und -intuition beruht und somit die Rationalität schwer zu überprüfen ist. Ebenso ziehen über $50 \%$ der Unternehmen für die Marketingbudgetfestlegung das Marketingbudget der Vorperiode heran, was einen vergangenheitsorientierten und wenig outputorientierten Fortschreibungsansatz impliziert. Differenzierte zielorientierte Methoden, die über Umsatz oder Absatz hinausgehen, sind relativ selten anzutreffen. Einen geringen Stellenwert nehmen beispielsweise branchen- bzw. wettbewerbsbezogene Größen für die Marketingbudgetierung (Abb. 4: branchenübliche Werte $=6,9 \%$, Marketingbudgets der Hauptwettbewerber $=1,9 \%)$ ein; dies dürfte nicht zuletzt darauf zurückzuführen sein, dass diese Werte außerhalb der marktforscherisch besonders gut erschlossenen Konsumgüterbranchen lediglich mit großem Aufwand erhältlich sind.

\section{Marketing- und Verkaufskennzahlen}

Des Weiteren wurde, wie in Abb. 5 illustriert, nach allen eingesetzten Marketingund Verkaufskennzahlen (grüner Balken) sowie den fünf wichtigsten Kennzahlen (grauer Balken) gefragt. Zur Messung der Effektivität und Effizienz des Marketing werden hauptsächlich absatz- und finanzwirtschaftliche Marketing- und Verkaufskennzahlen, wie zum Beispiel Umsatz, Nettogewinn und Umsatzrentabilität sowie der Absatz verwendet. Aber 
auch kundenorientierte Kennzahlen wie Kundenzufriedenheit, und wahrgenommene Kundenzufriedenheit werden gemessen, wenn auch seltener als TopSteuerungskennzahl eingesetzt. Konkurrenzorientierte Kenngrößen werden (mit Ausnahme des Marktanteils) kaum eingesetzt. Ferner lässt sich feststellen, dass auf komplexere sowie differenziertere Marketingkennzahlen wie Kunden- und Markenwert sowie Commitment eher selten zurückgegriffen wird.

Mittelwertvergleiche der Zufriedenheit mit dem Marketingcontrolling in Bezug auf die Verwendung der verschiedenen Kennzahlen zeigen, dass der Einsatz von komplexeren Kennzahlen signifikant mit einer höheren Zufriedenheit mit dem Marketingcontrolling einhergeht. So sind Unternehmen, die beispielsweise Kundenzufriedenheit(s-Index) sowie Kundenbindung(s-Index) messen, eindeutig zufriedener mit dem unternehmensinternen Marketingcontrolling als diejenigen Unternehmen, die auf diese Kennzahlen verzichten (Abb. 6).

Ähnliches zeigt sich bei der Untersuchung des Zusammenhangs von Zielerreichung und dem Einsatz einzelner Marketingkenngrößen: Unternehmen, die Kennzahlen zu Kundenzufriedenheit sowie Kundenbindung einsetzen, schätzen ihre (finanzwirtschaftliche sowie marktund kundengerichtete) Zielerreichung der letzten drei Jahren signifikant besser ein (Abb. 7). Ebenso geht die Messung der wahrgenommenen Service- sowie Produktqualität mit höherer Zielerreichung einher (Abb. 7).

Kennzahlen haben im Rahmen des Marketingcontrollings eine hohe Bedeutung, allerdings erlangen sie nur durch Vergleiche Aussagekraft (vgl. Siegwart/Reinecke/Sander, 2010, S. 13 ff.): entweder durch Zeitreihenanalysen oder durch Konkurrenzvergleiche. Besonders interessant ist, dass die konkurrenzorientierte Kennzahl "Share of Voice“ (eigener Anteil am Branchenwerbeaufwand) zwar lediglich von $10.4 \%$ der befragten Unternehmen erhoben wird (Abb. 5), jedoch diese wenigen Anwender im Mittel signifikant zufriedener mit der Qualität des eigenen Marketingcontrollings sind (Abb.6) sowie ihre Zielerreichung signifikant höher einschätzen (Abb. 7) als diejenigen Unternehmen, die den Share of Voice nicht als Marketingkontrollgröße einsetzen. „Sophistication“" und Differenzierung der Marketingkenn-

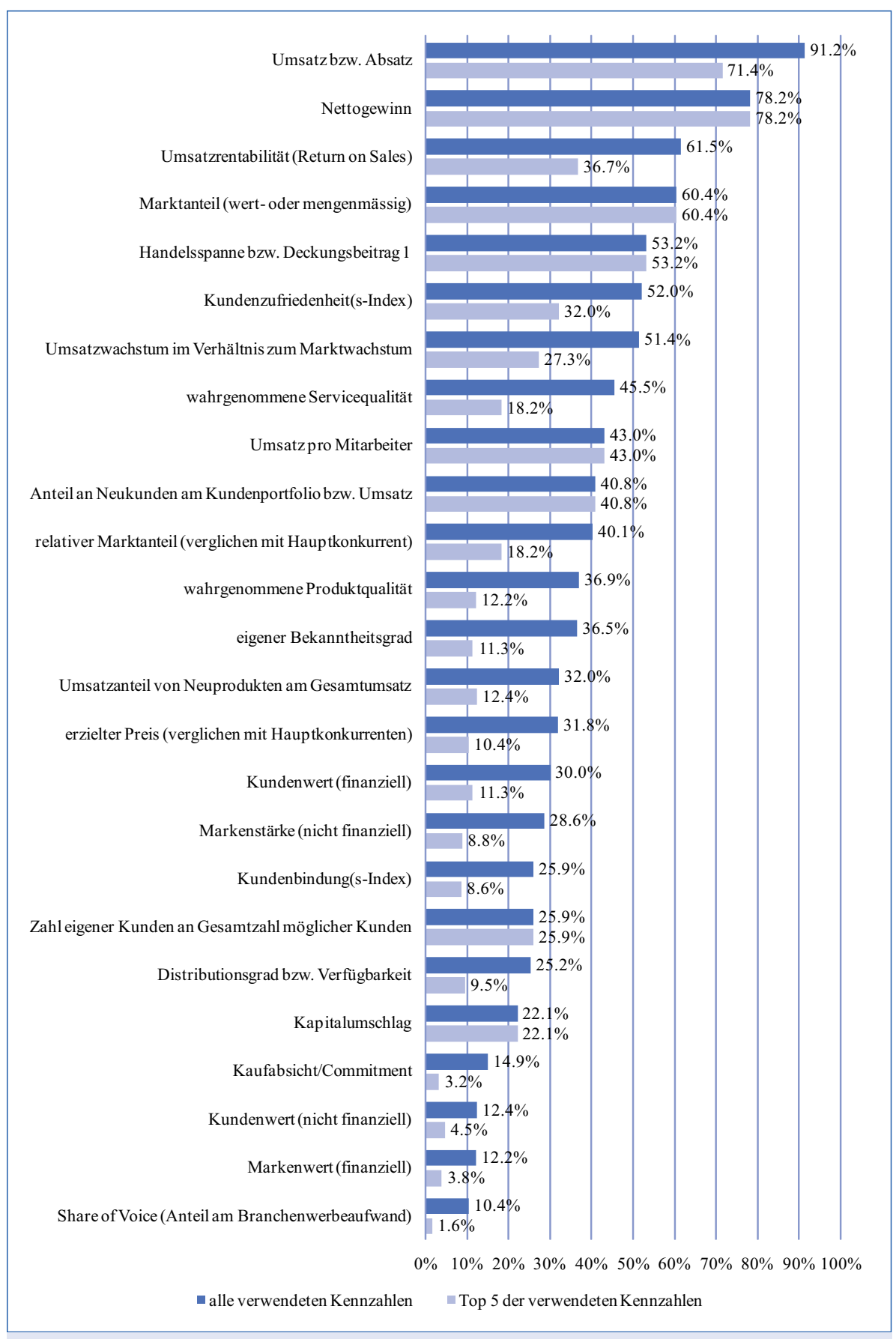

Abb. 5: Marketing- und Verkaufskennzahlen

zahlen einerseits und Marketingerfolg sowie Zufriedenheit andererseits gehen miteinander einher. (Aufgrund des Querschnittscharakters der vorliegenden Studie ist es allerdings nicht möglich, eine echte Kausalität zwischen diesen Konstrukten zu belegen. Zwar erscheint die Ursache-Wirkungsbeziehung zwischen Controllingintensität und Erfolg plausibel, doch wäre es durchaus denkbar, dass erfolgreiche Unternehmen intensiveres Marketingcontrolling betreiben, weil sie über die dafür erforderlichen finanziellen Mittel verfügen.)

\section{Instrumente des Marketingcontrollings}

Kennzahlen und Kennzahlensysteme sind lediglich ein Baustein eines umfassenden Marketingcontrolling-Systems; sie ergänzen, aber ersetzen keinesfalls Instrumente wie Deckungsbeitrags- oder Investitionsrechnung für Neuprodukteinführung. Gerade im Marketing gibt es zahlreiche Bereiche, die mit Kennzahlen nur unzureichend abgedeckt werden können (vgl. 


\begin{tabular}{|c|c|c|c|c|}
\hline \multirow[b]{3}{*}{ Kennzahl } & \multicolumn{4}{|c|}{ Zufriedenheit } \\
\hline & \multicolumn{2}{|c|}{ Mittelwert Zufriedenheit } & \multirow[b]{2}{*}{ Differenz } & \multirow[b]{2}{*}{$\begin{array}{l}* \text { signifikant }(\mathrm{p}<=0.05) \\
* * \text { sehr signifikant }(\mathrm{p}<=0.01) \\
* * * \text { hoch signifikant }(\mathrm{p}<=0.001)\end{array}$} \\
\hline & $\begin{array}{l}\text { Kennzahl } \\
\text { verwendet }\end{array}$ & $\begin{array}{l}\text { Kennzahl } \\
\text { nicht } \\
\text { verwendet }\end{array}$ & & \\
\hline $\begin{array}{l}\text { relativer Marktanteil } \\
\text { (verglichen mit } \\
\text { Hauptkonkurrent) }\end{array}$ & 3.96 & 3.63 & 0.329 & * \\
\hline $\begin{array}{l}\text { Umsatzwachstum im } \\
\text { Verhältnis zum } \\
\text { Marktwachstum }\end{array}$ & 3.94 & 3.58 & 0.359 & * \\
\hline $\begin{array}{l}\text { Zahl eigener Kunden an } \\
\text { Gesamtzahl möglicher } \\
\text { Kunden }\end{array}$ & 4.02 & 3.68 & 0.343 & $*$ \\
\hline $\begin{array}{l}\text { Anteil an Neukunden am } \\
\text { Kundenportfolio bzw. } \\
\text { Umsatz }\end{array}$ & 3.97 & 3.63 & 0.339 & $*$ \\
\hline $\begin{array}{l}\text { Kundenzufriedenheit(s- } \\
\text { Index) }\end{array}$ & 4.01 & 3.50 & 0.517 & $* * *$ \\
\hline Kundenbindung(s-Index) & 4.35 & 3.57 & 0.774 & $* * *$ \\
\hline Kundenwert (finanziell) & 4.07 & 3.64 & 0.430 & $* *$ \\
\hline $\begin{array}{l}\text { Kundenwert (nicht } \\
\text { finanziell) }\end{array}$ & 4.16 & 3.71 & 0.450 & * \\
\hline $\begin{array}{l}\text { Share of Voice (Anteil am } \\
\text { Branchenwerbeaufwand) }\end{array}$ & 4.30 & 3.70 & 0.601 & * \\
\hline $\begin{array}{l}\text { wahrgenommene } \\
\text { Produktqualität }\end{array}$ & 4.04 & 3.61 & 0.432 & $* *$ \\
\hline $\begin{array}{l}\text { wahrgenommene } \\
\text { Servicequalität }\end{array}$ & 3.98 & 3.59 & 0.396 & $* *$ \\
\hline Kaufabsicht/Commitment & 4.28 & 3.68 & 0.601 & $* *$ \\
\hline
\end{tabular}

Abb. 6: Mittelwertvergleich der Zufriedenheit in Bezug auf verwendete Kennzahlen

Reinecke, 2010, S. 81). Deshalb stellt sich die Frage, mit welchen Instrumenten und Verfahren Marketingführungskräfte versuchen, den Erfolg des Marketing zu steuern und zu belegen. Nachfolgend wird die Nutzung strategischer, operativer und finanzorientierter Marketingcontrolling-Instrumente dargestellt.

\section{Strategisches Marketingcontrolling}

Abb. 8 gibt einen Überblick zur Nutzung strategischer Marketingcontrolling-Instrumente. Zunächst ist anhand dieses Schaubilds offensichtlich, dass traditionelle Instrumente wie Konkurrenz-, Branchen-, Kundenzufriedenheits- und Mitarbeiterzufriedenheitsanalysen sowie Frühwarn- und Monitoringsysteme am häufigsten eingesetzt werden. Scheinbar setzt man bei wichtigen, strategischen Entscheidungen vermehrt auf bewährte Methoden. Auch wenn ein regelmäßiges Tracking der Kundenzufriedenheit sicherlich grundsätzlich sinnvoll ist und auch die Zufriedenheit mit dem Marketingcontrolling positiv beeinflusst, so stellt sich bei Kundenzufriedenheitsanalysen häufig die Frage nach dem Grenznutzen: In den meisten Branchen ändert sich die Kundenzufriedenheit nur sehr wenig, weil es sich um eine globale Einstellungsgröße handelt; bei trägen GröBen kann es daher aus wirtschaftlichen Gründen durchaus sinnvoll sein, die Erhebungsfrequenz zu reduzieren.

Hingegen wird die klassische Szenariotechnik äußerst unregelmäßig eingesetzt. Ein Grund hierfür dürfte die Subjektivität sowie der damit einhergehende wahrgenommene "esoterische Charakter" von Zukunftsprognosen sein. Erstaunlicherweise finden die in der wissenschaftlichen Literatur ausgiebig diskutierten Methoden wie die Scorecards oder Markenaudits in der Praxis nur vereinzelt Anwendung. Möglicherweise sind diese Methoden (noch) ,zu innovativ“ oder auch zu komplex, um sich einem breiteren Publikum zu erschließen.

Auch beim Thema Marktsegmentierung offenbart sich ein großer Gegensatz zwischen Wissenschaft und Praxis: Während die Notwendigkeit einer fundierten Marktsegmentierung in der Marketingliteratur weitgehend unstrittig ist, verzichten viele Unternehmen auf den regelmäBigen Einsatz von Segmentierungsunter- suchungen. Dies ist ein Indiz dafür, dass Unternehmen nach wie vor Schwierigkeiten haben, eine Segmentierung von der Planung bis zur Implementierung in Kommunikation und Verkauf erfolgreich umzusetzen (vgl. Belz, 2009, S. 79 f.).

\section{Controlling der Marketinginstrumente}

Das operative Marketingcontrolling (Abb. 9) bezieht sich primär auf die vier klassischen Marketinginstrumente: Marktleistungs- und Preisgestaltung, Kommunikation und Distribution.

Die Ergebnisse zeigen, dass im instrumentellen Bereich nachvollziehbarerweise das traditionell teuerste Marketinginstrument, der persönliche Verkauf, intensiv kontrolliert wird. Ferner wird eher auf traditionelle Verfahren gesetzt: Preis-, Produkt- oder Servicequalitätsanalysen finden nach wie vor regelmäßige Beachtung in der betriebswirtschaftlichen Praxis.

Die Kommunikationsforschung wird dagegen nicht sehr intensiv eingesetzt, was wahrscheinlich auf die relativ hohen Kosten, die erforderliche Methoden(beurteilungs)kompetenz sowie grundsätzliche Vorbehalte gegenüber der Möglichkeit eines Kommunikationscontrollings $\mathrm{zu}-$ rückzuführen ist. Werbepretests werden lediglich von $14,7 \%$ der befragten Unternehmen regelmäßig eingesetzt, Posttests nur von $16,7 \%$. Bedenkenswert ist, dass nur gerade $23,7 \%$ ein regelmäßiges Kommunikationstracking durchführen - zumal ein unregelmäßiges Tracking nicht Ziel führend und somit kaum sinnvoll ist.

Es zeigt sich somit, dass lediglich jene Instrumente des Marketingcontrollings stark zum Einsatz kommen, die ohnehin häufig aus Standardmarktforschungsstudien, aus dem Qualitätsmanagement oder aus finanzwirtschaftlichen Routinereports vorliegen. Auch werden kaum spezifische Maßnahmen zum Controlling des Marketing- bzw. Media-Mix getroffen; gerade dies wäre allerdings in Zeiten integrierter Kommunikation und CrossMedia-Kampagnen erforderlich.

Instrumente des Kosten- und Erfolgscontrollings haben einen direkten finanzwirtschaftlichen Bezug. Wie Abb. 10 zeigt, gehören Budgetanalyse (82,9\%), Deckungsbeitragsrechnungen bezüglich Produkten/Aufträgen/Projekten (71,2 \%) sowie Vollkosten- und Investitionsrechnungen (je 62,9\%) aus der Sicht der befragten Führungskräfte zu den Standard- 
instrumenten. Diese Instrumente des Marketingaccountings standen noch vor zwanzig Jahren im Zentrum des Marketingcontrollings, weil sie einen zentralen Informationsengpass betrafen; heutzutage liefern die informationstechnischen Controllinglösungen weitgehend zufriedenstellende Standardauswertungen. Dennoch offenbart sich eine gewisse Lücke: Die Marketingplanung ist in den letzten Jahren deutlich kunden(gruppen)orientierter geworden (vgl. Homburg/Droll/Tozek, 2008); dennoch werden kundenorientierte Deckungsbeitragsrechnungen bei deutlich weniger als der Hälfte der Unternehmen regelmäßig eingesetzt, was auf erhebliche Defizite des Marketingaccounting schließen lässt. Wie lässt sich kundenorientiertes Marketing planen und umsetzen, wenn das Controlling keine Informationen darüber liefert, welches die tatsächlich rentablen Kunden(gruppen) eines Unternehmens sind?

Für die in letzter Zeit intensiv diskutierten Markenwertberechnungen (13,1\%) scheint dagegen ebenso wie für Customer-Lifetime-Value-Analysen noch viel an Überzeugungsarbeit geleistet werden zu müssen. Anscheinend fehlt hier noch ein verlässlicher Methodenstandard, der den Anforderungen der Praxis gerecht wird. Positiv überrascht dagegen der von den Befragten angegebene relativ starke Einsatz von Prozesskostenrechnung und Target Costing.

Insgesamt scheint es im Marketingcontrolling eine Tendenz zu traditionellen, „bewährten“ Methoden zu geben. Neuere und methodisch anspruchsvollere Verfahren werden scheinbar als riskant, zu aufwändig oder zu teuer erachtet; oder aber es fehlt in der Unternehmenspraxis noch das entsprechende Know-how. Dennoch sind es gerade diese innovativen Marketingcontrollinginstrumente, welche eine Quantifizierung und Monetarisierung von Marketingmaßnahmen ermöglichen und Unternehmen dem Ziel einer wertorientierten Marketingführung näher bringen würden.

\section{Fazit}

Unternehmensvertreter sehen Marketing zwar grundsätzlich eher als Investition als „lediglich“ als Aufwand. Dennoch sind Führungskräfte mit dem Marketingcontrolling tendenziell eher unzufrieden, ob-

\begin{tabular}{|c|c|c|c|c|}
\hline \multirow[b]{3}{*}{ Kennzahl } & \multicolumn{4}{|c|}{ Zielerreichung } \\
\hline & \multicolumn{2}{|c|}{ Mittelwert Zielerreichung } & \multirow[b]{2}{*}{ Differenz } & \multirow[b]{2}{*}{$\begin{array}{l}\text { *signifikant }(\mathrm{p}<=0.05) \\
* * \text { sehr signifikant }(\mathrm{p}<=0.01) \\
\mathrm{z} * * \text { hoch signifikant }(\mathrm{p}<=0.001)\end{array}$} \\
\hline & $\begin{array}{l}\text { Kennzahl } \\
\text { verwendet }\end{array}$ & \begin{tabular}{|l|} 
Kennzahl \\
nicht \\
verwendet
\end{tabular} & & \\
\hline & \multicolumn{4}{|c|}{ Finanzwirtschaftliche Ziele } \\
\hline $\begin{array}{l}\text { Marktanteil (wert- oder } \\
\text { mengenmässig) }\end{array}$ & 5.48 & 5.07 & 0.415 & ** \\
\hline $\begin{array}{l}\text { relativer Marktanteil } \\
\text { (verglichen mit } \\
\text { Hauptkonkurrent) }\end{array}$ & 5.52 & 5.18 & 0.337 & * \\
\hline Nettogewinn & 5.40 & 4.99 & 0.416 & * \\
\hline $\begin{array}{l}\text { Handelsspanne bzw. } \\
\text { Deckungsbeitrag } 1\end{array}$ & 5.50 & 5.11 & 0.389 & * \\
\hline $\begin{array}{l}\text { Kundenzufriedenheit(s- } \\
\text { Index) }\end{array}$ & 5.60 & 5.01 & 0.595 & $* * *$ \\
\hline Kundenbindung(s-Index) & 5.59 & 5.22 & 0.364 & * \\
\hline Kundenwert (finanziell) & 5.58 & 5.20 & 0.381 & * \\
\hline $\begin{array}{l}\text { Kundenwert (nicht } \\
\text { finanziell) }\end{array}$ & 5.75 & 5.25 & 0.505 & * \\
\hline $\begin{array}{l}\text { Share of Voice (Anteil am } \\
\text { Branchenwerbeaufwand) }\end{array}$ & 5.84 & 5.25 & 0.589 & ** \\
\hline eigener Bekanntheitsgrad & 5.58 & 5.16 & 0.418 & $* *$ \\
\hline $\begin{array}{l}\text { wahrgenommene } \\
\text { Produktqualität }\end{array}$ & 5.57 & 5.17 & 0.398 & * \\
\hline $\begin{array}{l}\text { wahrgenommene } \\
\text { Servicequalität }\end{array}$ & 5.63 & 5.04 & 0.593 & $* * *$ \\
\hline \multirow[t]{2}{*}{\begin{tabular}{|l|} 
Distributionsgrad bzw. \\
Verfügbarkeit
\end{tabular}} & 5.73 & 5.17 & 0.554 & $* * *$ \\
\hline & \multicolumn{4}{|c|}{ Markt- und kundengerichtete Ziele } \\
\hline $\begin{array}{l}\text { Marktanteil (wert- oder } \\
\text { mengenmässig) }\end{array}$ & 5.17 & 4.85 & $0.320 *$ & $* * *$ \\
\hline $\begin{array}{l}\text { Kundenzufriedenheit(s- } \\
\text { Index) }\end{array}$ & 5.33 & 4.73 & $0.601 *$ & * \\
\hline Kundenbindung(s-Index) & 5.41 & 4.92 & $0.495 *$ & $*$ \\
\hline Kundenwert (finanziell) & 5.28 & 4.94 & $0.340 *$ & $*$ \\
\hline $\begin{array}{l}\text { Kundenwert (nicht } \\
\text { finanziell) }\end{array}$ & 5.38 & 4.99 & $0.386 *$ & $*$ \\
\hline $\begin{array}{l}\text { Share of Voice (Anteil am } \\
\text { Branchenwerbeaufwand) }\end{array}$ & 5.48 & 4.99 & 0.488 * & $*$ \\
\hline eigener Bekanntheitsgrad & 5.25 & 4.91 & 0.344 * & $* * *$ \\
\hline $\begin{array}{l}\text { wahrgenommene } \\
\text { Produktqualität }\end{array}$ & 5.22 & 4.94 & $0.286 *$ & $*$ \\
\hline $\begin{array}{l}\text { wahrgenommene } \\
\text { Servicequalität }\end{array}$ & 5.27 & 4.85 & $0.422 *$ & $* * *$ \\
\hline $\begin{array}{l}\text { Distributionsgrad bzw. } \\
\text { Verfügbarkeit }\end{array}$ & 5.32 & 4.95 & $0.374 *$ & * \\
\hline
\end{tabular}

\section{Abb. 7: Mittelwertvergleich Zielerreichung in Bezug auf verwendete Kennzahlen}

wohl dieses eine wesentliche Basis für ein Sicherstellen von Effektivität und Effizienz im Marketing ist. Dieser Sachverhalt führt zu der Annahme, dass Marketing nur sehr oberflächlich als Investition ausgegeben wird, in der Realität aber noch ein langer Weg zu einem wertorientierten Marketingmanagement bewältigt werden muss. Ursprung der Unzufriedenheit mit dem Marketingcontrolling scheinen die mangelnde Messbarkeit des Erfolgs von Marketingmaßnahmen, die unzureichen- de Informations- und Datenbasis sowie fehlende Fachkenntnisse zu sein.

In den letzten drei Jahren ist die Nachweispflicht des "Return on Marketing“ gegenüber dem Top-Management deutlich gestiegen. Das Berichtswesen ist allerdings noch stark geprägt von traditionellen, stark produktorientiertem finanzwirtschaftlichen Controlling. Der Einsatz prospektiver, kunden- und wertorientierter Accountingverfahren wie beispiels- 


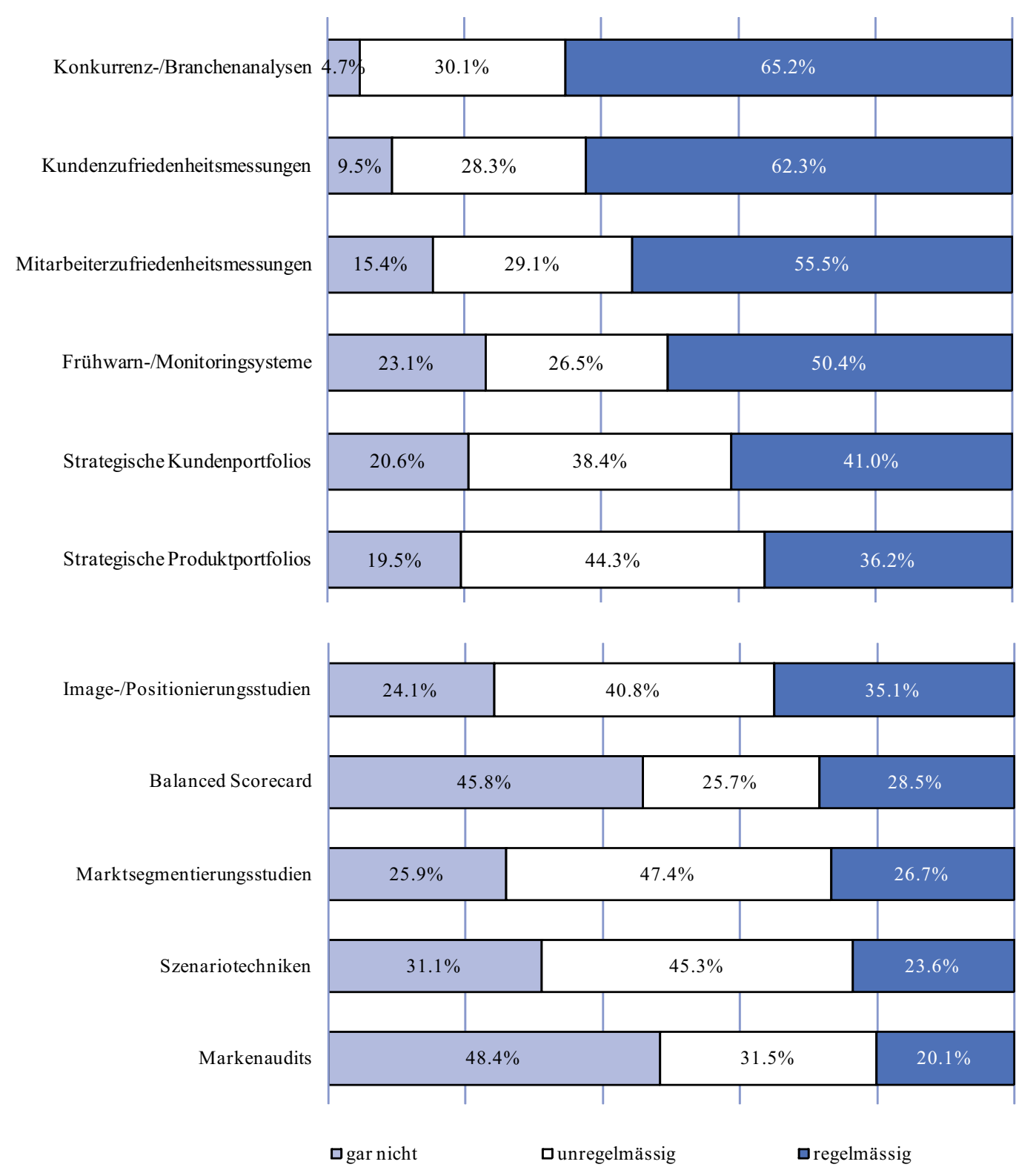

Abb. 8: Einsatz strategischer Marketingcontrolling-Instrumente

weise Customer-Value-Analysen sowie einem differenziertem, auf Marktforschung beruhendem Kommunikationscontrolling ist aufgrund der finanziellen und personellen Ressourcenbasis kaum realisierbar.

Hier erscheint ein Umdenken erforderlich: Forderungen nach einem verbesserten Reporting an das Top Management haben wenig Sinn, wenn diese nicht durch den Aufbau von Fach- und Methodenkompetenz begleitet werden. Planung ohne Controlling ist nur beschränkt sinnvoll - somit erscheint es angebracht, einen Mindestanteil des Marketingbudgets für eine Professionalisierung im Marketingcontrolling $\mathrm{zu}$ investieren, sofern auch hier vorgängig etwaige Effizienzreserven ausgeschöpft worden sind. Eine gewisse Investition knapper Marketingmittel zur Evaluation von Möglichkeiten zur Steigerung der Marketingeffektivität und -effizienz ist insbesondere dort sinnvoll, wo bisher weit gehend auf Feedback im Sinne eines Lernprozesses verzichtet wurde.

Handlungsbedarf besteht insbesondere bezüglich:

- der Professionalisierung der Marketingbudgetierung: Unternehmen sollten das Marketingbudget nicht primär prozentual vom Umsatz oder durch Fortschreibung bisheriger Budgets bestimmen, sondern vielmehr eindeutige Marketingereignisse und -ziele definieren (bspw. „Steigerung des ungestützten Bekanntheitsgrads bei der Zielgruppe der Jugendlichen um 5 Prozentpunkte im nächsten Jahr" oder „Erreichen eines Umsatzanteils von Neuprodukten von insgesamt $20 \%$ binnen der nächsten 2 Jahr"). Darauf aufbauend sind die entsprechenden Marketingmaßnahmen festzulegen und zu budgetieren, die erforderlich sind, um diese Ziele zu erreichen. 


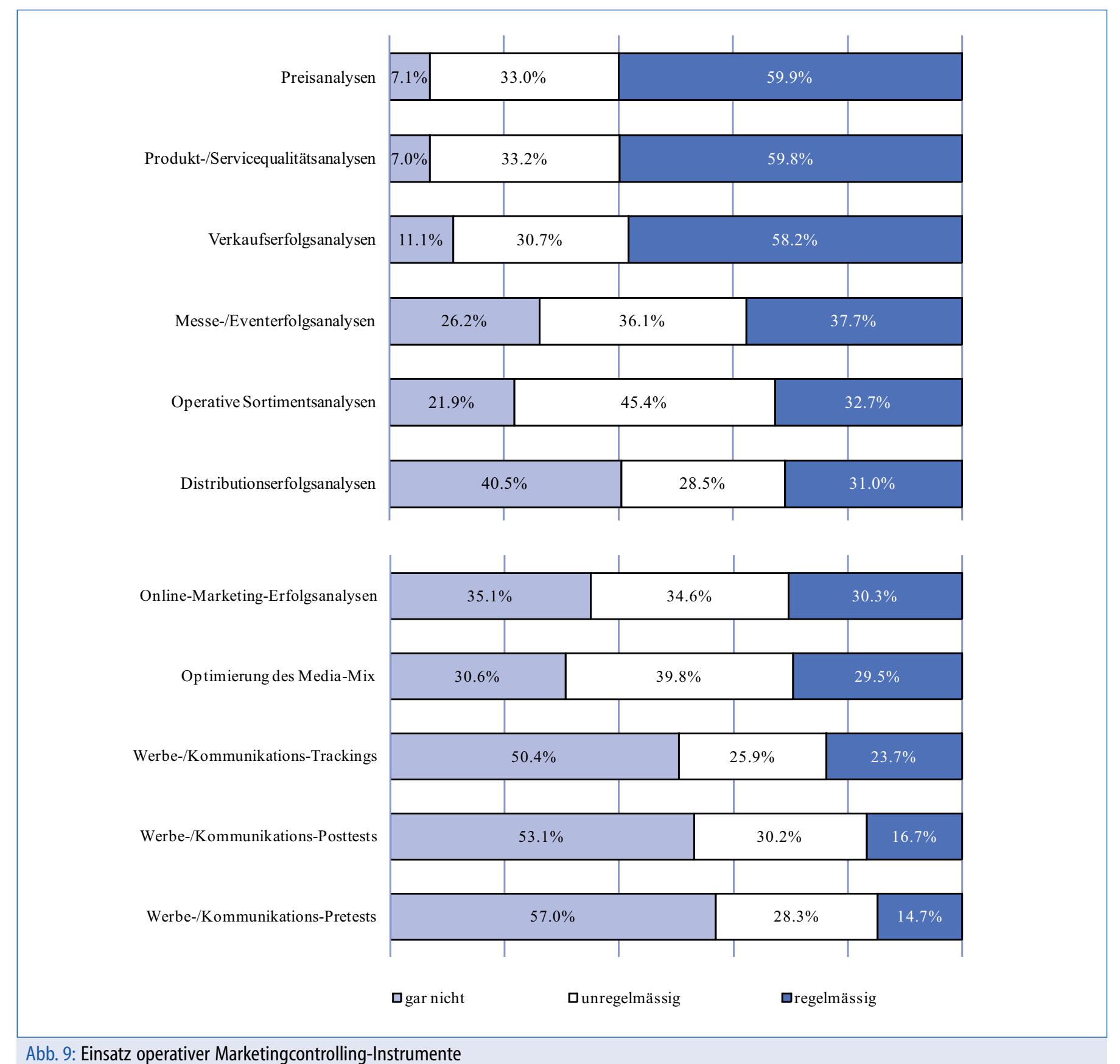

Abb. 9: Einsatz operativer Marketingcontrolling-Instrumente

- der Optimierung des Kommunikationscontrollings: Für jede zentrale Kommunikationsmaßnahme (z. B. Direct Mail, Messestand, Werbekampagne) sollten vorgängig klare Ziele gesetzt werden (z. B. erwarteter Rücklauf, Besuchsfrequenz, Image- und Bekanntheitsgrad). Diese Ziele sollten nachgängig mittels Marktforschung gemessen werden und Gründe für etwaige Abweichungen (sowohl positive als auch negative) analysiert werden, um daraus für die nächsten Kommunikationsmaßnahmen zu lernen. Bei- spielsweise steigern Unternehmen wie Procter \& Gamble und Dell die Prozesseffektivität und -effizienz durch Benchmarking erheblich.

Zusätzlich sollte bei aufwändigen Kommunikationsmaßnahmen zumindest das Verständnis der Werbebotschaft bei der Zielgruppe mittels Pretests geprüft werden.

- dem kunden(gruppen)orientierten Marketingaccounting: Auch wenn die Finanzsysteme der meisten Unternehmen auf Produkte bzw. Aufträge als Kostenträger ausgerichtet sind, so ist es insbesondere für Unternehmen im Beziehungsgeschäften unerlässlich, das Accounting vollumfänglich auch auf Kunden und Kundengruppen auszurichten. Eine mehrstufige Kundendeckungsbeitragsrechnung muss heutzutage als selbstverständliche Grundlage für ein professionelles Marketingmanagement angesehen werden.

Diese Maßnahmen würden die Wertund Zielorientierung des Marketing sowie die Zufriedenheit der Führungskräfte mit dem Marketingcontrolling bereits deutlich erhöhen. 


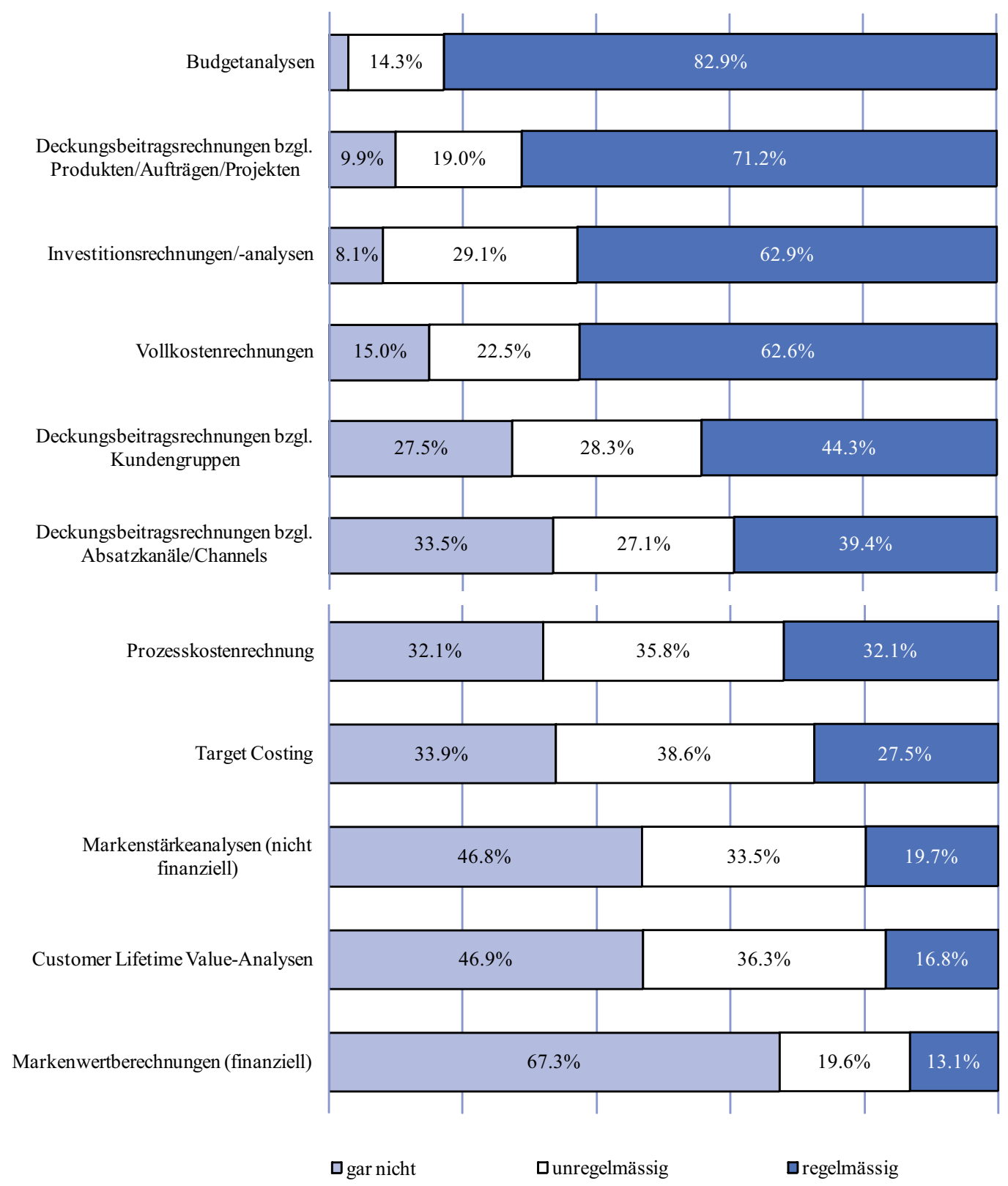

Abb. 10: Einsatz finanzorientierter Marketingcontrolling-Instrumente

Keywords
Marketing Controlling
Marketing Ratios
Marketing Budgeting
Marketing Controlling Instruments
Return on Marketing
Summary
The relevance of marketing control-
ling in business practice is undoubted:
Especially when economic faces chal-
lenging times, the marketing manage-
ment has to prove the effectiveness
and efficiency of spent marketing
budget. Scientist from the field of

marketing and controlling developed numerous of suggestions for effective marketing controlling. However, the gap between theoretic-conceptual development and the implementation of marketing controlling into daily business seems to be obvious. The following article is based on an empirical study, which demonstrates the actual use of marketing controlling instruments and methods. Following the results, areas of implementation issues as well as aspects requiring further research are discovered.

\section{Literatur}

Ambler, T., Marketing and the Bottom Line, 2. Aufl., Upper Saddle River 2003.

Bauer, H.H./Stokburger, G./Hammerschmidt, M., Marketing Performance, Wiesbaden 2006. $\mathrm{Belz}, \mathrm{C}$., Marketing gegen den Strom, St. Gallen 2009.

Farris, P.W./Bendle, N.T./Pfeifer, P.E./Reibstein, D.J., Marketing Metrics, 50+ Metrics Every Executive Should Master, Upper Saddle River (NJ) 2006.

Fuchs, D., Marketingbudgetierung: konzeptionelle Grundlagen und empirische Befunde, St. Gallen 2008. 
Homburg, C./Droll, M./Tozek, D., Customer Prioritization: Does It Pay Off, and How Should It Be Implemented?, in: Journal of Marketing, 72. Jg. (2008), H. 5, S. 110-130.

Link, J./Gerth, N./Voßbeck, E., MarketingControlling, München 2000.

Moorman, C./Lehmann, D. (Editor), Assessing Marketing Performance, Cambridge 2004.

O'Sullivan, D./Abela, A.V., Marketing Performance Measurement Ability and Firm Performance, in: Journal of Marketing, 71. Jg. (2007), H. 2, S. 79-93.

Reinecke, S., Marketing Performance Management: Empirisches Fundament und Konzeption für ein integriertes Marketingkennzahlensystem, Wiesbaden 2004.

Reinecke, S./Janz, S., Marketingcontrolling: Sicherstellen von Effektivität und Effizienz des Marketing, Stuttgart 2007.

Reinecke, S., Marketingcontrolling, Sicherstellen von Effektivität und Effizienz einer marketingorientierten Unternehmensführung, in: Controlling, 22. Jg. (2010), Heft 2, S. 75-82.

Rust, R.T./Ambler, T./Carpenter, G.S./Kumar, V./Srivastava, R.K., Measuring Marketing Productivity: Current Knowledge and Future Directions, in: Journal of Marketing, 68. Jg. (2004), October, S. 76-89.

Sheth, J.N./Sisodia, R.S., Feeling the Heat, in: Marketing Management, 4. Jg. (1995), H. 2, S. 9-23.

Sheth, J.N./Sisodia, R.S., Marketing Productivity - Issues and Analysis, in: Journal of Business Research, 55. Jg. (2002), S. 349-362.

Siegwart, H./Reinecke, S./Sander, S., Kennzahlen für die Unternehmensführung, 7. Aufl., Bern 2010.

Weber, J., Managing the Marketing Budget in a Cost-Constrained Environment, in: Industrial Marketing Management, 31. Jg. (2002), S. 705-717.

\section{Controlling Praxis}

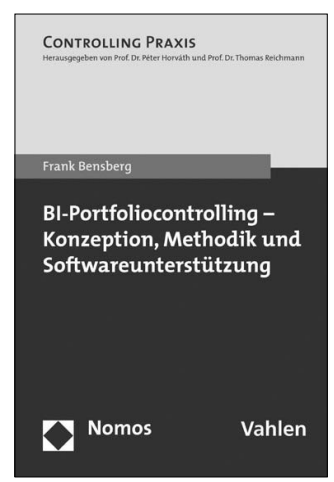

BI-Portfoliocontrolling-Konzeption, Methodik und Softwareunterstützung Von PD Dr. Frank Bensberg 2010, 303 S., brosch., 59,- $€$, ISBN 978-3-8329-5253-2

Business Intelligence-Systeme sind für Unternehmen ein wichtiges Mittel zur Erzielung von Wettbewerbsvorteilen. Zur systematischen Umsetzung dieser Vorteile liefert dieses Werk eine innovative, praxistaugliche Methodik, die an den Entscheidungsprozessen betrieblicher Akteure ansetzt. Diese Prozesse werden im Rahmen eines Portfolioansatzes analysiert, um Gestaltungsmaßnahmen für BI-Systeme abzuleiten.

\section{Kosten- und Erlösrechnung unter unscharfer Sicherheit}

Fuzzifizierung der Grenzplankostenrechnung Von Dr. Marcus Roso

2009, 312 S., brosch., 49,- $€$ ISBN 978-3-8329-4866-5

Unscharfe Mengen werden in diesem Buch auf die Kosten- und Erlösrechnung angewandt. Aufbauend auf einer Systematisierung von Informationsständen wird die unscharfe Mengenlehre als Instrument zur Abbildung von Unsicherheit - insbesondere im Rahmen der Grenzplankostenrechnung - identifiziert. Die Vorteile werden anhand eines Fallbeispiels dargelegt.

\section{Internationale Printmedien-Joint Ventures \\ Wachstumsoption und Steuerungsherausforderung Von Prof. Dr. Boris Alexander Kühnle}

2009, 417 S., brosch., 69,-€, ISBN 978-3-8329-3685-3

Wachstum durch Internationalisierung - immer mehr Verlage wenden diese Formel an. Lokales Know-How ist dafür unabdingbar, weshalb Printmedien-Unternehmen häufig internationale Joint Ventures zum Markteintritt wählen. Wie aber lassen sich Joint Ventures erfolgreich steuern? Die Arbeit gibt Antworten auf Basis der Untersuchung internationaler und Joint Ventureerfahrener Verlage.

\section{Nomos}

